\title{
Identification of differentially expressed genes in human bladder cancer through genome-wide gene expression profiling
}

\author{
KAZUMORI KAWAKAMI $^{1,3}$, HIDEKI ENOKIDA ${ }^{1}$, TOKUSHI TACHIWADA ${ }^{1}$, \\ TAKENARI GOTANDA ${ }^{1}$, KENGO TSUNEYOSHI ${ }^{1}$, HIROYUKI KUBO $^{1}$, KENRYU NISHIYAMA $^{1}$, \\ MASAKI TAKIGUCHI ${ }^{2}$, MASAYUKI NAKAGAWA ${ }^{1}$ and NAOHIKO SEKI ${ }^{3}$
}

${ }^{1}$ Department of Urology, Graduate School of Medical and Dental Sciences, Kagoshima University, 8-35-1 Sakuragaoka, Kagoshima 890-8520; Departments of ${ }^{2}$ Biochemistry and Genetics, and ${ }^{3}$ Functional Genomics, Graduate School of Medicine, Chiba University, 1-8-1 Inohana, Chuo-ku, Chiba 260-8670, Japan

Received February 15, 2006; Accepted April 27, 2006

\begin{abstract}
Large-scale gene expression profiling is an effective strategy for understanding the progression of bladder cancer (BC). The aim of this study was to identify genes that are expressed differently in the course of $\mathrm{BC}$ progression and to establish new biomarkers for BC. Specimens from 21 patients with pathologically confirmed superficial $(n=10)$ or invasive $(\mathrm{n}=11) \mathrm{BC}$ and 4 normal bladder samples were studied; samples from 14 of the $21 \mathrm{BC}$ samples were subjected to microarray analysis. The validity of the microarray results was verified by real-time RT-PCR. Of the 136 up-regulated genes we detected, 21 were present in all 14 BCs examined (100\%), 44 in 13 (92.9\%), and the other 71 in 12 BCs (85.7\%). Of 69 down-regulated genes, 25 were found in all 14 BCs (100\%), 22 in 13 (92.9\%), and the other 22 in 12 BCs (85.7\%). Functional annotation revealed that of the up-regulated genes, $36 \%$ were involved in metabolism and $14 \%$ in transcription and processing; $25 \%$ of the down-regulated genes were linked to cell adhesion/surface and $21 \%$ to cytoskeleton/cell membrane. Real-time RT-PCR confirmed the microarray results obtained for the 6 most highly up- and the 2 most highly down-regulated genes. Among the 6 most highly upregulated genes, $C K S 2$ was the only gene with a significantly greater level of up-regulation in invasive than in superficial $\mathrm{BC}(\mathrm{p}=0.04)$. To confirm this result, we subjected all $21 \mathrm{BC}$ samples to real-time PCR assay for $C K S 2$. We found a considerable difference between superficial and invasive $\mathrm{BC}$ $(\mathrm{p}=0.001)$. Interestingly, there was a considerable difference between the normal bladder and invasive $\mathrm{BC}(\mathrm{p}=0.001)$ and less difference between the normal bladder and superficial $\mathrm{BC}(\mathrm{p}=0.005)$. We identified several genes as promising candidates for diagnostic biomarkers of human $\mathrm{BC}$ and the
\end{abstract}

Correspondence to: Dr Hideki Enokida, Department of Urology, Graduate School of Medical and Dental Sciences, Kagoshima University, 8-35-1 Sakuragaoka, Kagoshima 890-8520, Japan

E-mail: enokida@m.kufm.kagoshima-u.ac.jp

Key words: microarray, expression profile, bladder cancer, $C K S 2$
CKS2 gene not only as a potential biomarker for diagnosing, but also for staging human $\mathrm{BC}$. This is the first report demonstrating that $C K S 2$ expression is strongly correlated with the progression of human $\mathrm{BC}$.

\section{Introduction}

Bladder cancer (BC) is among the 5 most common malignancies worldwide, and the 2 nd most common tumor of the genitourinary tract and the 2 nd most common cause of death in patients with cancer of the urinary tract (1-7). At diagnosis, BC with progression usually appears to be superficial (pT1); $20 \%$ of tumors with muscle invasion at the time of diagnosis tend to progress rapidly and their prognosis is unfavorable (3-5). The ability to predict, at the first biopsy, whether a BC shift to progression is probable would facilitate the selection of appropriate treatment modalities and improve the prognosis of patients with this cancer. Due to their insufficient sensitivity and specificity, none of the biomarkers now available for the diagnosis of $\mathrm{BC}$ can replace cystoscopy or cytology $(3,6,7)$ and patients with suspected $\mathrm{BC}$ continue to be subjected to painful cystoscopy.

Gene expression profiling has been used in the molecular classification of many tumor types. Molecular subtypes with potential diagnostic and prognostic implications have been identified (3,8-10). DNA microarrays aid in the outcome prediction for cancer patients because they facilitate the simultaneous analysis of the expression profiles of thousands of genes, making possible the identification of groups of genes with different expression profiles in tumors related to different outcomes. These gene-expression profiles assist in the selection of optimum treatment strategies by allowing therapies to be precisely adapted to different types of tumors (8-10).

Microarray analysis have identified cancer-related genes in $\mathrm{BC}(3,4,11-15)$. For example, the p33 inhibitor of the growth family 1 (p33ING1) and cathepsin E (CTSE) expression level, are correlated with the progression and prognosis of BC $(11,14)$. While these studies provided useful insights into the molecular biology of BC, their sensitivity and specificity are limited and their usefulness for 
Table I. Patient characteristics.

\begin{tabular}{rrrrr}
\hline No. & Age & Gender & Stage & Grade \\
\hline 1 & 55 & F & superficial & G2 \\
2 & 61 & M & superficial & G2 \\
3 & 68 & M & superficial & G2 \\
4 & 68 & M & superficial & G2 \\
5 & 88 & M & invasive & G3 \\
6 & 80 & M & invasive & G3 \\
7 & 53 & F & superficial & G2 \\
8 & 85 & M & superficial & G2 \\
9 & 53 & M & superficial & G2 \\
10 & 78 & M & invasive & G3 \\
11 & 83 & M & invasive & G2 \\
12 & 74 & F & superficial & G2 \\
13 & 89 & M & superficial & G3 \\
14 & 70 & M & invasive & G3 \\
15 & 47 & F & superficial & G2 \\
16 & 71 & M & invasive & G3 \\
17 & 75 & M & invasive & G3 \\
18 & 87 & F & invasive & G2 \\
19 & 76 & M & invasive & G3 \\
20 & 59 & M & invasive & G3 \\
21 & 78 & M & invasive & G2 \\
\hline & & & &
\end{tabular}

predicting disease outcome remains unclear (13-15). Using gene expression analysis and DNA microarrays, we looked for novel gene clusters related to human BC in an attempt to discover new biomarkers.

\section{Materials and methods}

Tissue samples. Tissue samples were obtained from 21 patients with BC (10 superficial, 11 invasive) who had undergone cystectomy or transurethral resection of bladder tumors at Kagoshima University Hospital, Kagoshima, Japan (Table I). Each tumor was staged and graded according to the TNM staging system (16) and the Japanese Urological Association and the Japanese Society of Pathology (17). Our study was approved by the Bioethics Committee of Kagoshima University; written prior informed consent was obtained from all patients for use of their samples and clinical and pathological data.

Sample preparation and total RNA extraction. Freshly harvested tissues, immediately frozen in liquid nitrogen and stored at $-80^{\circ} \mathrm{C}$, were dissolved in TRIzol reagent (Invitrogen, Carlsbad, CA, USA); for total RNA extraction we followed the manufacturer's protocol. We used premium total RNA (from normal human bladder; Clontech, Palo Alto, CA, USA) as a reference for microarray analysis. RNA density was measured in an Ultrospec 3100 Pro instrument (Amersham Biosciences), RNA quality was checked in an Agilent 2100 bioanalyzer (Agilent Technologies).

Antisense RNA (aRNA) amplification. For microarray analysis we used good-quality total RNA samples from 9 patients with superficial- and 5 patients with invasive BC (samples 1-14, Table I). aRNA was amplified from $5 \mu \mathrm{g}$ total RNA using the amino allyl message Amp aRNA amplification kit (Ambion, Austin, TX, USA). We amplified single-strand cDNA using T7 oligos (dT), converted the product into double-stranded cDNA, purified this cDNA, and then performed amplification from double-strand cDNA templates using the manufacturer's protocol.

Dye coupling and microarray hybridization. Oligoarrays, AceGene ${ }^{\circledR}$ human oligo chip $30 \mathrm{~K}$ (http://bio.hitachi-sk. co.jp/acegene/index.html, Hitachi Software Engineering Co. Ltd., Yokohama, Japan) spotted with $\sim 30,144$ genes, were used for dye coupling (normal bladder -Cy3, BC -Cy5) and microarray hybridization. Pellets were formed with ethanolprecipitated aRNA $(5 \mu \mathrm{g})$ and $5 \mu \mathrm{l}$ CyDye (Amersham Bioscience); $5 \mathrm{x}$ fragmentation buffer was added after purification and following further refinement, we obtained concentrated coupled aRNA. A hybridization solution was added to the microarrays and this was followed by $18-\mathrm{h}$ incubation at $50^{\circ} \mathrm{C}$.

cDNA preparation and quantitative real-time RT-PCR. Total RNA $(2 \mu \mathrm{g})$ was mixed with $0.5 \mu \mathrm{g}$ of oligo-dT primer and $0.4 \mu \mathrm{l}$ of dNTPs $(25 \mathrm{mM})$; a final volume of $25 \mu \mathrm{l}$ was prepared for single-strand synthesis. Using the manufacturer's protocol, we prepared $21 \mathrm{cDNA}$ samples from the same total RNA used for microarray analysis $(n=14)$ and from 7 additional BCs. For normal bladder controls we prepared 4 cDNA samples from 3 different lots of premium total RNA (human normal bladder, Clontech) and human bladder total RNA (Chemicon International, Inc., Temecula, CA, USA). Gene-specific PCR products were assayed continuously using a 7300 Real-Time PCR system (Applied Biosystems, Foster City, CA, USA) according to the manufacturer's protocol. Briefly, the initial PCR step was a 10 min hold at $95^{\circ} \mathrm{C}$; the cycles $(n=40)$ consisted of a $15-\mathrm{sec}$ denaturation step at $95^{\circ} \mathrm{C}$ followed by 1 -min annealing/extension at $63^{\circ} \mathrm{C}$. Primers used for real-time PCR were as follows: FABP5: 5'ttggttcagcatcaggagtg-3' (sense), 5'-cctgtccaaagtgatg atgg-3' (antisense); PABPC1: 5'-agccatgcaccctactctg-3' (sense), 5'tctttagcttggtgggcttg-3' (antisense); CKS2: 5'-catgagccagaa ccacatattc-3' (sense), 5'-cagctcatgcacaggtatgg-3' (antisense); SF3B1: 5'-ccctagagggcctgagagtt-3' (sense), 5'-tgtgctatgagagc gtcctg-3' (antisense); DDX5: 5'-tcccaagttgcttcagttgg-3' (sense), 5'-ccttttgcccgcagagtatc-3' (antisense); EIF3S6: 5'cttggtggcttgtcttgagg-3' (sense), 5'-atcttggcatccagtcttgc-3' (antisense); TAGLN: 5'-aagaatgatgggcactaccg-3' (sense), 5'actgatgatctgccgaggtc-3' (antisense); TPM2: 5'-actgga caacgcactcaatg-3' (sense), 5'-gttggcaatttctgctcctc-3' (antisense); RPL37A: 5'-taccaagaaagtcgggatcg-3' (sense), 5'tcttcatgcaggaaccacag-3' (antisense). All reactions were performed in duplicate and a negative control lacking cDNA was included. The gene encoding ribosomal protein L37a (RPL37A) served as an internal control because in our hands it showed the smallest Cy5/Cy3 fluctuation. RPL37A located on chromosome $2 \mathrm{q} 35 \mathrm{q}$ is quite different from $R P L 37$ located on chromosome 5p13 (Table II). The relative mRNA expression examined was normalized to the amount of RPL37A in the same cDNA using the standard curve method provided by the manufacturer. 
Table II. Frequently up-regulated genes in bladder cancer.

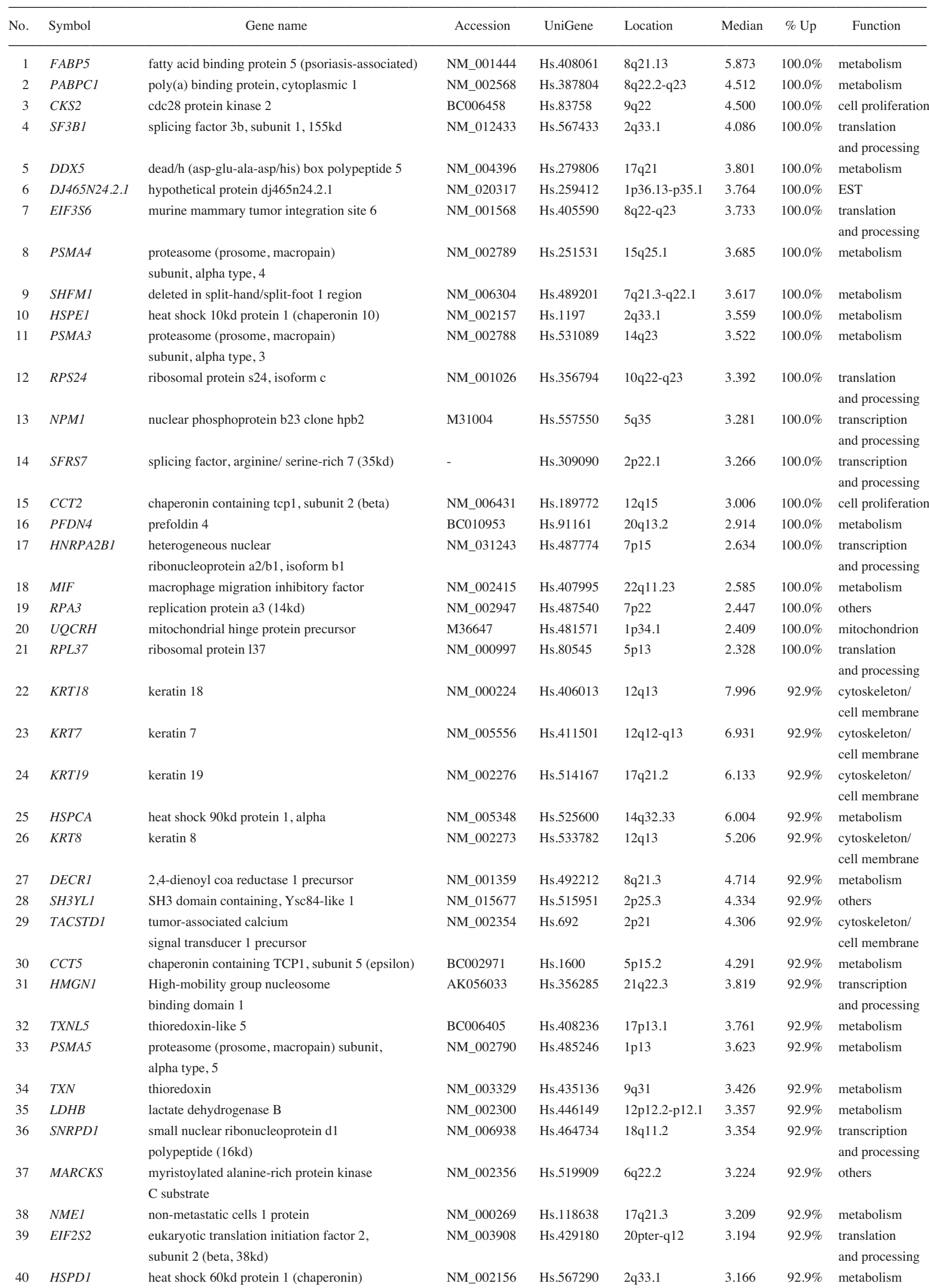


Table II. Continued.

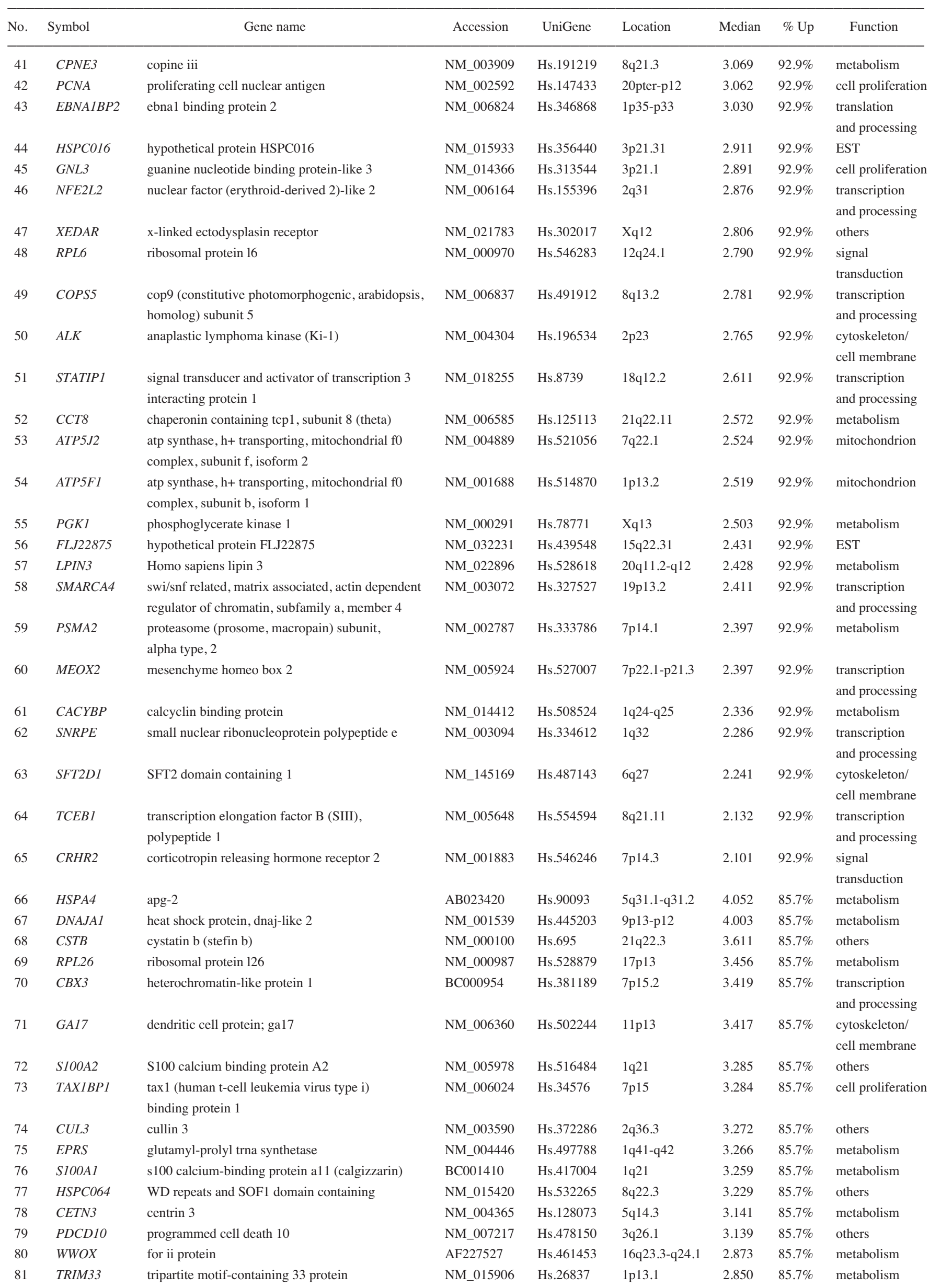


Table II. Continued.

\begin{tabular}{|c|c|c|c|c|c|c|c|c|}
\hline No. & Symbol & Gene name & Accession & UniGene & Location & Median & $\% \mathrm{Up}$ & Function \\
\hline 82 & - & ensembl genscan prediction & AL163932 & - & chromosome 14 & 2.846 & $85.7 \%$ & EST \\
\hline 83 & $P P$ & pyrophosphatase (inorganic) & NM_021129 & Hs. 437403 & 10q11.1-q24 & 2.837 & $85.7 \%$ & metabolism \\
\hline 84 & CYCS & cytochrome c & NM_018947 & Hs.437060 & $7 \mathrm{p} 15.3$ & 2.792 & $85.7 \%$ & mitochondrion \\
\hline 85 & FXYD3 & fxyd domain-containing ion transport regulator 3 & ВT006712 & Hs. 301350 & $19 \mathrm{q} 13.11-\mathrm{q} 13.12$ & 2.769 & $85.7 \%$ & metabolism \\
\hline 86 & USP47 & ubiquitin specific peptidase 47 & NM_017944 & Hs. 567521 & $11 \mathrm{p} 15.3$ & 2.760 & $85.7 \%$ & metabolism \\
\hline 88 & PSMD14 & 26 s proteasome-associated pad 1 homolog & NM_005805 & Hs. 567410 & $2 \mathrm{q} 24.2$ & 2.724 & $85.7 \%$ & metabolism \\
\hline 89 & C13orf12 & chromosome 13 open reading frame 12 & NM_015932 & Hs. 268742 & $13 q 12.3$ & 2.698 & $85.7 \%$ & others \\
\hline 90 & $R B 1$ & retinoblastoma 1 & NM_000321 & Hs .408528 & $13 q 14.2$ & 2.691 & $85.7 \%$ & cell proliferation \\
\hline 91 & $A T P 2 B 1$ & atpase, $\mathrm{ca}^{++}$transporting, plasma membrane 1 & NM_001682 & Hs. 506276 & $12 \mathrm{q} 21.3$ & 2.690 & $85.7 \%$ & metabolism \\
\hline 92 & LOC554202 & hypothetical LOC554202 & BC011715 & Hs. 458096 & $9 \mathrm{p} 21.3$ & 2.687 & $85.7 \%$ & EST \\
\hline 94 & KIAA0220 & KIAA0220-like protein & XM_290670 & Hs. .531664 & $16 \mathrm{p} 12.3$ & 2.659 & $85.7 \%$ & others \\
\hline 95 & - & ensembl genscan prediction & AC026900 & - & chromosome 1 & 2.654 & $85.7 \%$ & EST \\
\hline 96 & NEK6 & putative serine-threonine protein kinase & NM_014397 & Hs.197071 & $9 q 33.3-q 34.11$ & 2.616 & $85.7 \%$ & metabolism \\
\hline 97 & FIBL-6 & weakly similar to fibulin-1 isoform d precursor & AK027344 & Hs. 58877 & $1 q 25.3-q 31.1$ & 2.612 & $85.7 \%$ & metabolism \\
\hline 98 & RPS7 & ribosomal protein s7 & NM_001011 & Hs. 546287 & $2 \mathrm{p} 25$ & 2.588 & $85.7 \%$ & $\begin{array}{l}\text { translation } \\
\text { and processing }\end{array}$ \\
\hline 99 & - & ensembl genscan prediction & AL031186 & - & $22 q 12.1-12.2$ & 2.565 & $85.7 \%$ & EST \\
\hline 100 & $S R I$ & sorcin & NM_003130 & Hs. 489040 & $7 \mathrm{q} 21.1$ & 2.552 & $85.7 \%$ & metabolism \\
\hline 101 & FLJ22662 & hypothetical protein flj22662 & NM_024829 & Hs. 131933 & $12 \mathrm{p} 13.1$ & 2.541 & $85.7 \%$ & EST \\
\hline 102 & $R A N$ & ras-related nuclear protein & NM_006325 & Hs.10842 & $12 \mathrm{q} 24.3$ & 2.534 & $85.7 \%$ & cell proliferation \\
\hline 103 & DC6 & dc6 protein & NM_020189 & Hs. 492555 & $8 \mathrm{q} 23.1$ & 2.531 & $85.7 \%$ & $\begin{array}{l}\text { transcription } \\
\text { and processing }\end{array}$ \\
\hline 104 & VPS35 & vacuolar protein sorting 35 (yeast) & NM_018206 & Hs. 454528 & $16 \mathrm{q} 12$ & 2.519 & $85.7 \%$ & metabolism \\
\hline 106 & KIAA1542 & CTD-binding SR-like protein rA9 & NM_020901 & Hs. 325838 & $11 \mathrm{p} 15.5$ & 2.489 & $85.7 \%$ & metabolism \\
\hline 107 & HSPC111 & hypothetical protein HSPC111 & NM_016391 & Hs. 529475 & $5 \mathrm{q} 35.2$ & 2.488 & $85.7 \%$ & $\begin{array}{l}\text { intracellular } \\
\text { organelle }\end{array}$ \\
\hline 108 & SCFD1 & sec 1 family domain containing 1 & NM_016106 & Hs. 369168 & $14 q 12$ & 2.479 & $85.7 \%$ & metabolism \\
\hline 109 & $P I G L$ & phosphatidylinositol glycan, class 1 & NM_004278 & Hs.499793 & $17 \mathrm{p} 12-\mathrm{p} 11.2$ & 2.465 & $85.7 \%$ & metabolism \\
\hline 110 & LSM3 & LSM3 homolog, U6 small nuclear RNA associated & NM_014463 & Hs.111632 & $3 \mathrm{p} 25.1$ & 2.454 & $85.7 \%$ & $\begin{array}{l}\text { transcription } \\
\text { and processing }\end{array}$ \\
\hline 111 & WDR61 & WD repeat domain 61 & AF309553 & Hs.513055 & $15 q 25.1$ & 2.451 & $85.7 \%$ & $\begin{array}{l}\text { transcription } \\
\text { and processing }\end{array}$ \\
\hline 112 & LOC389651 & similar to hypothetical protein (L1H 3 region) & XM_372039 & Hs. 567978 & $8 \mathrm{p} 11.1$ & 2.434 & $85.7 \%$ & EST \\
\hline 113 & LEPRE1 & growth suppressor 1 & NM_022356 & Hs.437656 & $1 \mathrm{p} 34.1$ & 2.424 & $85.7 \%$ & others \\
\hline 114 & - & ensembl genscan prediction & AC011371 & - & chromosome 5 & 2.402 & $85.7 \%$ & EST \\
\hline 115 & GNGT2 & $\begin{array}{l}\text { guanine nucleotide binding protein-gamma } \\
\text { transducing activity polypeptide } 2\end{array}$ & NM_031498 & Hs. 181781 & $17 \mathrm{q} 21$ & 2.393 & $85.7 \%$ & $\begin{array}{l}\text { signal } \\
\text { transduction }\end{array}$ \\
\hline 116 & $N R B F 2$ & nuclear receptor binding factor 2 & NM_030759 & Hs. 449628 & $10 \mathrm{q} 21.3$ & 2.388 & $85.7 \%$ & $\begin{array}{l}\text { transcription } \\
\text { and processing }\end{array}$ \\
\hline 117 & $A R P C 2$ & actin related protein $2 / 3$ complex, subunit 2 & NM_005731 & Hs. 529303 & $2 \mathrm{q} 36.1$ & 2.380 & $85.7 \%$ & $\begin{array}{l}\text { cytoskeleton/ } \\
\text { cell membrane }\end{array}$ \\
\hline 118 & SEC61G & sec61 gamma & NM_014302 & Hs. 488282 & $7 \mathrm{p} 11.2$ & 2.378 & $85.7 \%$ & $\begin{array}{l}\text { intracellular } \\
\text { organelle }\end{array}$ \\
\hline 119 & $S S B P 1$ & single-stranded dna-binding protein & NM_003143 & Hs.490394 & $7 \mathrm{q} 34$ & 2.369 & $85.7 \%$ & mitochondrion \\
\hline 120 & C14orf112 & chromosome 14 open reading frame 112 & NM_016468 & Hs. 137108 & $14 \mathrm{q} 24.2$ & 2.367 & $85.7 \%$ & others \\
\hline 121 & ATP5E & $\begin{array}{l}\text { atp synthase, } h+\text { transporting, mitochondrial } \\
\text { f1 complex, epsilon subunit }\end{array}$ & NM_006886 & Hs. 177530 & $20 \mathrm{q} 13.32$ & 2.362 & $85.7 \%$ & mitochondrion \\
\hline 122 & ETFA & electron transfer flavoprotein, alpha polypeptide & NM_000126 & Hs.39925 & $15 q 23-q 25$ & 2.345 & $85.7 \%$ & metabolism \\
\hline 123 & $T B X 4$ & t-box 4 & NM_018488 & Hs.143907 & $17 q 21-q 22$ & 2.328 & $85.7 \%$ & $\begin{array}{l}\text { transcription } \\
\text { and processing }\end{array}$ \\
\hline
\end{tabular}


Table II. Continued.

\begin{tabular}{|c|c|c|c|c|c|c|c|c|}
\hline No. & Symbol & Gene name & Accession & UniGene & Location & Median & $\% \mathrm{Up}$ & Function \\
\hline 126 & ZNF84 & zinc finger protein 84 (HPF2) & NM_003428 & Hs. 445019 & $12 \mathrm{q} 24.33$ & 2.293 & $85.7 \%$ & $\begin{array}{l}\text { transcription } \\
\text { and processing }\end{array}$ \\
\hline 127 & LOC400451 & $\begin{array}{l}\text { hypothetical gene supported by AK075564; } \\
\text { BC } 060873\end{array}$ & NM_207446 & Hs. 27373 & $15 \mathrm{q} 26.1$ & 2.279 & $85.7 \%$ & EST \\
\hline 128 & MAPK $8 I P 2$ & $\begin{array}{l}\text { mitogen-activated protein kinase } 8 \text { interacting } \\
\text { protein } 2\end{array}$ & NM_012324 & Hs. 356523 & $22 \mathrm{q} 13.33$ & 2.261 & $85.7 \%$ & $\begin{array}{l}\text { signal } \\
\text { transduction }\end{array}$ \\
\hline 129 & $\mathrm{MDH} 2$ & malate dehydrogenase 2 , nad (mitochondrial) & NM_005918 & Hs. 520967 & $7 \mathrm{p} 12.3-\mathrm{q} 11.2$ & 2.202 & $85.7 \%$ & mitochondrion \\
\hline 130 & RNF39 & ring finger protein 39 & AF238317 & Hs. 121178 & $6 \mathrm{p} 21.3$ & 2.159 & $85.7 \%$ & metabolism \\
\hline 131 & $U C R C$ & ubiquinol-cytochrome $\mathrm{c}$ reductase complex & NM_013387 & Hs. 284292 & 22 cen-q12.3 & 2.153 & $85.7 \%$ & mitochondrion \\
\hline 132 & CSE1L & cse 1 chromosome segregation 1-like (yeast) & NM_001316 & Hs. 90073 & $20 \mathrm{q} 13$ & 2.127 & $85.7 \%$ & mitochondrion \\
\hline 133 & C6orf49 & chromosome 6 open reading frame 49 & NM_013397 & Hs.525899 & $6 \mathrm{p} 21.31$ & 2.064 & $85.7 \%$ & metabolism \\
\hline 134 & PSMA7 & $\begin{array}{l}\text { proteasome (prosome, macropain) subunit, } \\
\text { alpha type, } 7\end{array}$ & NM_002792 & Hs.233952 & $20 \mathrm{q} 13.33$ & 2.063 & $85.7 \%$ & metabolism \\
\hline 135 & C8orf17 & chromosome 8 open reading frame 17 & AF220264 & Hs. 283098 & $8 \mathrm{q} 24.3$ & 2.023 & $85.7 \%$ & cell proliferation \\
\hline 136 & SERP1 & stress-associated endoplasmic reticulum protein 1 & NM_014445 & Hs.518326 & $3 \mathrm{q} 25.1$ & 2.009 & $85.7 \%$ & $\begin{array}{l}\text { intracellular } \\
\text { organelle }\end{array}$ \\
\hline
\end{tabular}

Statistical analysis and annotation of gene function. Relationships between the 2 groups and the numerical values obtained by real-time RT-PCR were analyzed by the MannWhitney U test. Relationships among the 3 groups and the numerical values were analyzed by the Bonferroni-adjusted Mann-Whitney U test. The analysis software was Expert StatView (version 4, SAS Institute Inc., Cary, NC, USA); for comparison tests among the 3 groups, the non-adjusted statistical level of significance $(\mathrm{p}<0.05)$ corresponds to a Bonferroni-adjusted statistical significance of $\mathrm{p}<0.0167$.

The molecular function of the up- and down-regulated genes was classified into 13 groups as referenced in GENEONTOLOGY (http://www.geneontology.org/) and GeneCards (http://www.genecards.org/index.shtml), i.e. metabolism, transcription and processing, translation and processing, signal transduction, cell proliferation, cell-cycle regulation, cell differentiation, cell adhesion/surface-linked, cytoskeleton/cell membrane-linked, intracellular organelle, mitochondrion, other, and expressed sequence tags (ESTs) (18).

\section{Results}

Identification of genes expressed differently in BC and normal bladder. By microarray analysis of 14 BCs we identified 136 genes that were generally up-regulated more than 1.5 -fold in BC compared to normal bladder (Table II). Among these, 21 were up-regulated in all $14 \mathrm{BCs}(100 \%), 44$ in $13(92.9 \%)$, and the other 71 were up-regulated in 12 BCs $(85.7 \%)$. On the contrary, 69 genes were down-regulated less than -1.5-fold (Table III). Among these, 25 were down-regulated in all 14 BCs $(100 \%), 22$ in $13(92.9 \%)$, and the other 22 in 12 BCs $(85.7 \%)$.

Molecular function of up- and down-regulated genes in BC. The functional annotation of the 136 up- and 69 downregulated genes is presented in Fig. 1. Functionally, the upregulated genes were involved in metabolism (36\%), trans-

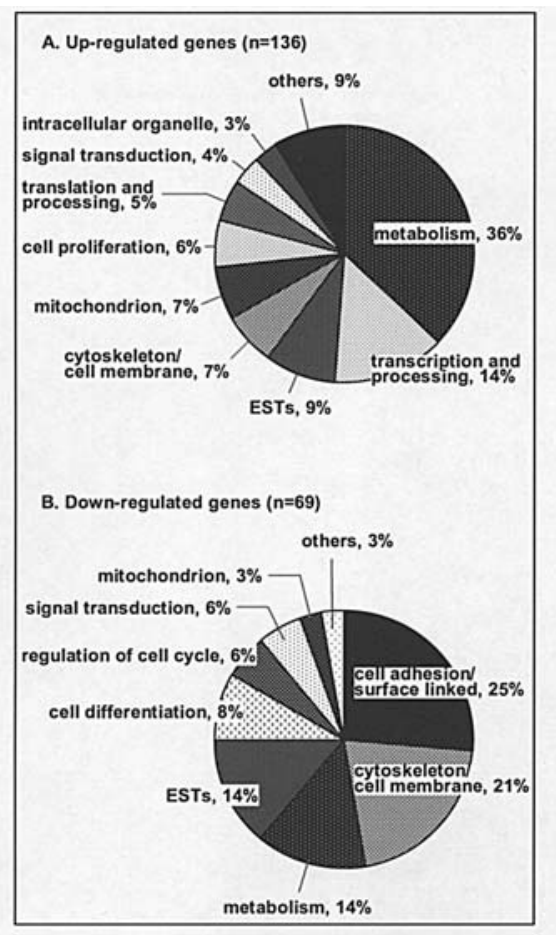

Figure 1. Distribution of the expression of functionally categorized genes in human BC. The functional features of the 136 up- (A) and 69 downregulated genes $(\mathrm{B})$ were classified into 13 categories.

cription and processing (14\%), ESTs (9\%), cytoskeleton/cell membrane $(7 \%)$, mitochondrion $(7 \%)$, cell proliferation $(6 \%)$, translation and processing (5\%), signal transduction $(4 \%)$, intracellular organelle (3\%), and other functions $(9 \%)$ (Fig. $1 \mathrm{~A})$. The down-regulated genes were involved in cell adhesion/surface linked (25\%), cytoskeleton/cell membrane (21\%), metabolism (14\%), ESTs (14\%), cell differentiation $(8 \%)$, cell-cycle regulation $(6 \%)$, signal transduction $(6 \%)$, mito-chondrion (3\%), and other functions (3\%) (Fig. 1B). 
Table III. Frequently down-regulated genes in bladder cancer.

\begin{tabular}{|c|c|c|c|c|c|c|c|c|}
\hline No. & Symbol & Gene name & Accession & UniGene & Location & Median & $\%$ Up Down & Function \\
\hline 1 & $T A G L N$ & transgelin & NM_003186 & Hs.503998 & $11 \mathrm{q} 23.2$ & 0.095 & $100.0 \%$ & $\begin{array}{l}\text { cytoskeleton/ } \\
\text { cell membrane }\end{array}$ \\
\hline 2 & TPM2 & tropomyosin 2 (beta) & NM_003289 & Hs. 300772 & $9 \mathrm{p} 13.2-\mathrm{p} 13.1$ & 0.113 & $100.0 \%$ & $\begin{array}{l}\text { cytoskeleton/ } \\
\text { cell membrane }\end{array}$ \\
\hline 3 & $M G P$ & matrix Gla protein & NM_000900 & Hs.365706 & 12p13.1-p12.3 & 0.121 & $100.0 \%$ & $\begin{array}{l}\text { cell adhesion/ } \\
\text { surface linked }\end{array}$ \\
\hline 4 & $G S N$ & gelsolin & NM_198252 & Hs. 522373 & $9 q 33$ & 0.147 & $100.0 \%$ & $\begin{array}{l}\text { cytoskeleton/ } \\
\text { cell membrane }\end{array}$ \\
\hline 5 & CNN1 & calponin 1 , basic, smooth muscle & NM_001299 & Hs.465929 & $19 \mathrm{p} 13.2-\mathrm{p} 13.1$ & 0.161 & $100.0 \%$ & $\begin{array}{l}\text { cytoskeleton/ } \\
\text { cell membrane }\end{array}$ \\
\hline 6 & $M Y L K$ & myosin light chain kinase, isoform 6 & NM_005965 & Hs. 477375 & $3 q 21$ & 0.164 & $100.0 \%$ & metabolism \\
\hline 7 & $D F$ & adipsin/complement factor $\mathrm{d}$ precursor & NM_001928 & Hs. 155597 & $19 \mathrm{p} 13.3$ & 0.168 & $100.0 \%$ & metabolism \\
\hline 8 & $T P M 1$ & tropomyosin 1 (alpha) & AL050179 & Hs.133892 & $15 q 22.1$ & 0.170 & $100.0 \%$ & $\begin{array}{l}\text { cytoskeleton/ } \\
\text { cell membrane }\end{array}$ \\
\hline 9 & FN1 & fibronectin 1 & NM_002026 & Hs. 203717 & $2 q 34$ & 0.171 & $100.0 \%$ & $\begin{array}{l}\text { cell adhesion/ } \\
\text { surface linked }\end{array}$ \\
\hline 10 & DES & desmin & NM_001927 & Hs. 471419 & $2 q 35$ & 0.176 & $100.0 \%$ & $\begin{array}{l}\text { cytoskeleton/ } \\
\text { cell membrane }\end{array}$ \\
\hline 11 & $I L K$ & integrin-linked kinase & NM_004517 & Hs. .5158 & $11 \mathrm{p} 15.5-\mathrm{p} 15.4$ & 0.192 & $100.0 \%$ & $\begin{array}{l}\text { signal } \\
\text { transduction }\end{array}$ \\
\hline 12 & $D C N$ & decorin & NM_001920 & Hs. 156316 & $12 \mathrm{q} 21.33$ & 0.206 & $100.0 \%$ & $\begin{array}{l}\text { cell adhesion/ } \\
\text { surface linked }\end{array}$ \\
\hline 13 & $C K B$ & creatine kinase, brain & NM_001823 & Hs. 173724 & $14 \mathrm{q} 32$ & 0.206 & $100.0 \%$ & metabolism \\
\hline 14 & MYL9 & myosin regulatory light chain 2 , smooth muscle isoform & NM_006097 & Hs. .504687 & $20 \mathrm{q} 11.23$ & 0.206 & $100.0 \%$ & $\begin{array}{l}\text { cytoskeleton/ } \\
\text { cell membrane }\end{array}$ \\
\hline 15 & $F B L N 5$ & fibulin 5 & NM_006329 & Hs. 332708 & $14 \mathrm{q} 32.1$ & 0.236 & $100.0 \%$ & $\begin{array}{l}\text { cell adhesion/ } \\
\text { surface linked }\end{array}$ \\
\hline 16 & $D S T N$ & destrin (actin depolymerizing factor) & NM_006870 & Hs.304192 & $20 \mathrm{p} 12.1$ & 0.244 & $100.0 \%$ & $\begin{array}{l}\text { cell adhesion/ } \\
\text { surface linked }\end{array}$ \\
\hline 17 & $S F R P 2$ & secreted frizzled-related protein 2 & BC008666 & Hs. 481022 & $4 \mathrm{q} 31.3$ & 0.250 & $100.0 \%$ & $\begin{array}{l}\text { regulation } \\
\text { of cell cycle }\end{array}$ \\
\hline 18 & FXYD6 & FXYD domain containing ion transport regulator 6 & NM_022003 & Hs. .504031 & $11 \mathrm{q} 23.3$ & 0.262 & $100.0 \%$ & $\begin{array}{l}\text { cytoskeleton/ } \\
\text { cell membrane }\end{array}$ \\
\hline 19 & $C R Y A B$ & crystallin, alpha b & NM_001885 & Hs. 408767 & $11 \mathrm{q} 22.3-\mathrm{q} 23.1$ & 0.274 & $100.0 \%$ & others \\
\hline 20 & $I G F B P 7$ & insulin-like growth factor binding protein 7 & NM_001553 & Hs. 479808 & $4 q 12$ & 0.276 & $100.0 \%$ & $\begin{array}{l}\text { regulation } \\
\text { of cell cycle }\end{array}$ \\
\hline 21 & COL6A2 & type vi collagen alpha 2 chain precursor & AY029208 & Hs. 420269 & $21 \mathrm{q} 22.3$ & 0.334 & $100.0 \%$ & $\begin{array}{l}\text { cell adhesion/ } \\
\text { surface linked }\end{array}$ \\
\hline 22 & $L G A L S 1$ & lectin, galactoside-binding, soluble, 1 (galectin 1) & ВC001693 & Hs. 445351 & $22 \mathrm{q} 13.1$ & 0.335 & $100.0 \%$ & $\begin{array}{l}\text { cell } \\
\text { differentiation }\end{array}$ \\
\hline 23 & $B C R$ & $\begin{array}{l}\text { bcr-abl mrna 5' fragment clone } 3 \mathrm{c} \text {; } \\
\text { unknown protein } 77 \text { aa }\end{array}$ & $\mathrm{X} 14675$ & Hs. 517461 & $22 q 11$ & 0.335 & $100.0 \%$ & $\begin{array}{l}\text { regulation } \\
\text { of cell cycle }\end{array}$ \\
\hline 24 & $M G L L$ & monoglyceride lipase & NM_007283 & Hs. 277035 & $3 q 21.3$ & 0.356 & $100.0 \%$ & metabolism \\
\hline 25 & HSPCO72 & HSPC072 protein & NM_014162 & Hs.439352 & $20 \mathrm{p} 11.23$ & 0.407 & $100.0 \%$ & EST \\
\hline 26 & $C A V 1$ & caveolin 1 & NM_001753 & Hs.74034 & $7 q 31.1$ & 0.180 & $92.9 \%$ & $\begin{array}{l}\text { cytoskeleton/ } \\
\text { cell membrane }\end{array}$ \\
\hline 27 & $C L E C 3 B$ & C-type lectin domain family 3 , member B & NM_003278 & Hs. 476092 & $3 \mathrm{p} 22-\mathrm{p} 21.3$ & 0.206 & $92.9 \%$ & $\begin{array}{l}\text { cytoskeleton/ } \\
\text { cell membrane }\end{array}$ \\
\hline 28 & COL1A2 & alpha 2 type i collagen preproprotein & NM_000089 & Hs. 489142 & $7 q 22.1$ & 0.211 & $92.9 \%$ & $\begin{array}{l}\text { cell adhesion/ } \\
\text { surface linked }\end{array}$ \\
\hline 29 & $C L U$ & clusterin & NM_001831 & Hs. 436657 & $8 \mathrm{p} 21-\mathrm{p} 12$ & 0.230 & $92.9 \%$ & EST \\
\hline 30 & $P L A 2 G 2 A$ & phospholipase a2, group iia (platelets, synovial fluid) & NM_000300 & Hs. 466804 & $1 \mathrm{p} 35$ & 0.247 & $92.9 \%$ & metabolism \\
\hline 31 & COL3A1 & alpha 1 type iii collagen preproprotein & NM_000090 & Hs. 443625 & $2 q 31$ & 0.266 & $92.9 \%$ & $\begin{array}{l}\text { cell adhesion/ } \\
\text { surface linked }\end{array}$ \\
\hline 32 & COL1A1 & collagen, type I, alpha 1 & NM_000088 & Hs. 172928 & $17 q 21.3-q 22.1$ & 0.274 & $92.9 \%$ & $\begin{array}{l}\text { cell adhesion/ } \\
\text { surface linked }\end{array}$ \\
\hline 33 & $I G F B P 6$ & insulin-like growth factor binding protein 6 & NM_002178 & Hs. 274313 & $12 q 13$ & 0.295 & $92.9 \%$ & $\begin{array}{l}\text { regulation } \\
\text { of cell cycle }\end{array}$ \\
\hline
\end{tabular}


Table III. Continued.

\begin{tabular}{|c|c|c|c|c|c|c|c|c|}
\hline No. & Symbol & Gene name & Accession & UniGene & Location & Median & $\%$ Up Down & Function \\
\hline 34 & $H B A 1$ & hemoglobin, alpha 1 & AF281258 & Hs .449630 & $16 \mathrm{p} 13.3$ & 0.296 & $92.9 \% \mathrm{n}$ & mitochondrion \\
\hline 35 & FHL1 & four and a half lim domains 1 & NM_001449 & Hs.435369 & $\mathrm{Xq} 26$ & 0.308 & $92.9 \%$ & $\begin{array}{l}\text { cytoskeleton/ } \\
\text { cell membrane }\end{array}$ \\
\hline 36 & PDLIM3 & alpha-actinin-2-associated lim protein & NM_014476 & Hs.85862 & $4 q 35$ & 0.317 & $92.9 \%$ & $\begin{array}{l}\text { cytoskeleton/ } \\
\text { cell membrane }\end{array}$ \\
\hline 37 & $A E B P 1$ & adipocyte enhancer binding protein 1 precursor & NM_001129 & Hs. 439463 & $7 \mathrm{p} 13$ & 0.350 & $92.9 \%$ & $\begin{array}{l}\text { cell adhesion/ } \\
\text { surface linked }\end{array}$ \\
\hline 38 & $C 1 R$ & complement component $1, \mathrm{r}$ subcomponent & NM_001733 & Hs.524224 & $12 \mathrm{p} 13$ & 0.376 & $92.9 \%$ & metabolism \\
\hline 39 & PCP4 & purkinje cell protein 4 & NM_006198 & Hs.80296 & $21 \mathrm{q} 22.2$ & 0.222 & $85.7 \%$ & metabolism \\
\hline 40 & CTSK & cathepsin k (pycnodysostosis) & NM_000396 & Hs.523594 & $1 \mathrm{q} 21$ & 0.386 & $92.9 \%$ & $\begin{array}{l}\text { cell adhesion/ } \\
\text { surface linked }\end{array}$ \\
\hline 41 & ACTG1 & actin, gamma 1 & ВC004223 & Hs.514581 & $17 \mathrm{q} 25$ & 0.416 & $92.9 \%$ & $\begin{array}{l}\text { cytoskeleton/ } \\
\text { cell membrane }\end{array}$ \\
\hline 42 & $P T G D S$ & prostaglandin $\mathrm{d} 2$ synthase ( $21 \mathrm{kd}$, brain) & NM_000954 & Hs. 446429 & $9 \mathrm{q} 34.2-\mathrm{q} 34.3$ & 0.418 & $92.9 \%$ & metabolism \\
\hline 43 & PRSS23 & putative secreted protein zsig 13 & AF193611 & Hs. 25338 & $11 \mathrm{q} 14.1$ & 0.421 & $92.9 \%$ & EST \\
\hline 44 & CKIP-1 & ck2 interacting protein $1 ;$ hq0024c protein; loc51177 & NM_016274 & Hs.438824 & $1 \mathrm{q} 21.2$ & 0.435 & $92.9 \%$ & EST \\
\hline 45 & TIMP1 & tissue inhibitor of metalloproteinase 1 precursor & NM_003254 & Hs.522632 & Xp11.3-p11.23 & 0.453 & $92.9 \%$ & $\begin{array}{l}\text { cell adhesion/ } \\
\text { surface linked }\end{array}$ \\
\hline 46 & $K L F 3$ & Kruppel-like factor 3 (basic) & NM_016531 & Hs. 298658 & $4 \mathrm{p} 14$ & 0.520 & $92.9 \%$ & $\begin{array}{l}\text { signal } \\
\text { transduction }\end{array}$ \\
\hline 47 & - & ensembl genscan prediction & AC007346 & - & chromosome 16 & 0.466 & $92.9 \%$ & EST \\
\hline 48 & МYH11 & myosin, heavy polypeptide 11 , smooth muscle & NM_022844 & Hs. 460109 & 16p13.13-p13.12 & 0.266 & $85.7 \%$ & $\begin{array}{l}\text { celladhesion/ } \\
\text { surface linked }\end{array}$ \\
\hline 49 & $I T M 2 A$ & integral membrane protein $2 \mathrm{a}$ & NM_004867 & Hs.17109 & $\mathrm{Xq} 13.3-\mathrm{Xq} 21.2$ & 0.318 & $85.7 \%$ & $\begin{array}{l}\text { cell } \\
\text { differentiation }\end{array}$ \\
\hline 50 & - & alpha heavy chain & $\mathrm{X} 17116$ & - & $14 \mathrm{q} 32$ & 0.323 & $85.7 \%$ & EST \\
\hline 51 & $R G S 2$ & regulator of g-protein signalling $2,24 \mathrm{kd}$ & NM_002923 & Hs.78944 & $1 \mathrm{q} 31$ & 0.355 & $85.7 \%$ & $\begin{array}{l}\text { signal } \\
\text { transduction }\end{array}$ \\
\hline 52 & COL6A2 & collagen, type VI, alpha 2 & ВC002484 & Hs.420269 & $21 \mathrm{q} 22.3$ & 0.370 & $85.7 \%$ & $\begin{array}{l}\text { cell adhesion/ } \\
\text { surface linked }\end{array}$ \\
\hline 53 & $V W F$ & von willebrand factor & NM_000552 & Hs. 440848 & $12 \mathrm{p} 13.3$ & 0.376 & $85.7 \%$ & $\begin{array}{l}\text { cell adhesion/ } \\
\text { surface linked }\end{array}$ \\
\hline 54 & $F B L N 1$ & fibulin 1 isoform d & NM_006486 & Hs. 24601 & $22 \mathrm{q} 13.31$ & 0.376 & $85.7 \%$ & $\begin{array}{l}\text { cell adhesion/ } \\
\text { surface linked }\end{array}$ \\
\hline 55 & SERPINF1 & $\begin{array}{l}\text { serine (or cysteine) proteinase inhibitor, } \\
\text { clade } f \text { member } 1\end{array}$ & NM_002615 & Hs. 532768 & $17 \mathrm{p} 13.1$ & 0.409 & $85.7 \%$ & $\begin{array}{l}\text { cell } \\
\text { differentiation }\end{array}$ \\
\hline 56 & - & nc_001807 mitochondrion complete genome & NC_001807 & - & mitochondrion & 0.414 & $85.7 \%$ & mitochondrion \\
\hline 57 & EFEMP1 & $\begin{array}{l}\text { egf-containing fibulin-like extracellular matrix } \\
\text { protein } 1 \text {, isoform b }\end{array}$ & NM_018894 & Hs.76224 & $2 \mathrm{p} 16$ & 0.425 & $85.7 \%$ & $\begin{array}{l}\text { cell adhesion/ } \\
\text { surface linked }\end{array}$ \\
\hline 58 & COX7A1 & $\begin{array}{l}\text { cytochrome c oxidase subunit viia polypeptide } 1 \\
\text { (muscle) }\end{array}$ & NM_001864 & Hs. 421621 & $19 \mathrm{q} 13.1$ & 0.431 & $85.7 \%$ & metabolism \\
\hline 59 & CSRP1 & cysteine and glycine-rich protein 1 & NM_004078 & Hs. 108080 & $1 \mathrm{q} 32$ & 0.433 & $85.7 \%$ & $\begin{array}{l}\text { cell } \\
\text { differentiation }\end{array}$ \\
\hline 60 & $H B D$ & hemoglobin, delta & NM_000519 & Hs.36977 & $11 \mathrm{p} 15.5$ & 0.451 & $85.7 \%$ & metabolism \\
\hline 61 & RPS6KA5 & rsk-like protein kinase rlpk & AF080000 & Hs. 510225 & $14 \mathrm{q} 31-\mathrm{q} 32.1$ & 0.465 & $85.7 \%$ & $\begin{array}{l}\text { signal } \\
\text { transduction }\end{array}$ \\
\hline 62 & $S P R R 3$ & small proline-rich protein 3 & AJ243667 & Hs.139322 & $1 \mathrm{q} 21-\mathrm{q} 22$ & 0.483 & $85.7 \%$ & $\begin{array}{l}\text { cell } \\
\text { differentiation }\end{array}$ \\
\hline 63 & $T B X 1$ & t-box 1 transcription factor $\mathrm{c}$ & AF373867 & Hs.173984 & $22 \mathrm{q} 11.21$ & 0.484 & $85.7 \%$ & others \\
\hline 64 & - & ensembl genscan prediction & AF277315 & - & $\mathrm{Xq} 28$ & 0.484 & $85.7 \%$ & EST \\
\hline 65 & $S P A R C$ & secreted protein, acidic, cysteine-rich (osteonectin) & NM_003118 & Hs.111779 & $5 q 31.3-q 32$ & 0.484 & $85.7 \%$ & $\begin{array}{l}\text { cell adhesion/ } \\
\text { surface linked }\end{array}$ \\
\hline 66 & - & ensembl genscan prediction & AC007601 & - & chromosome 16 & 0.509 & $85.7 \%$ & EST \\
\hline 67 & $S V I L$ & supervillin, isoform 1 & NM_003174 & Hs.499209 & 0.526 & $85.7 \%$ & cell & \\
\hline 68 & KIAA0582 & kiaa0582 protein & NM_015147 & Hs.146007 & $2 \mathrm{p} 14$ & 0.536 & $85.7 \%$ & $\begin{array}{l}\text { differentiation } \\
\text { EST }\end{array}$ \\
\hline 69 & - & hypothetical protein FLJ20186 & NM_207514 & Hs.62771 & $16 \mathrm{q} 24.3$ & 0.565 & $85.7 \%$ & EST \\
\hline
\end{tabular}




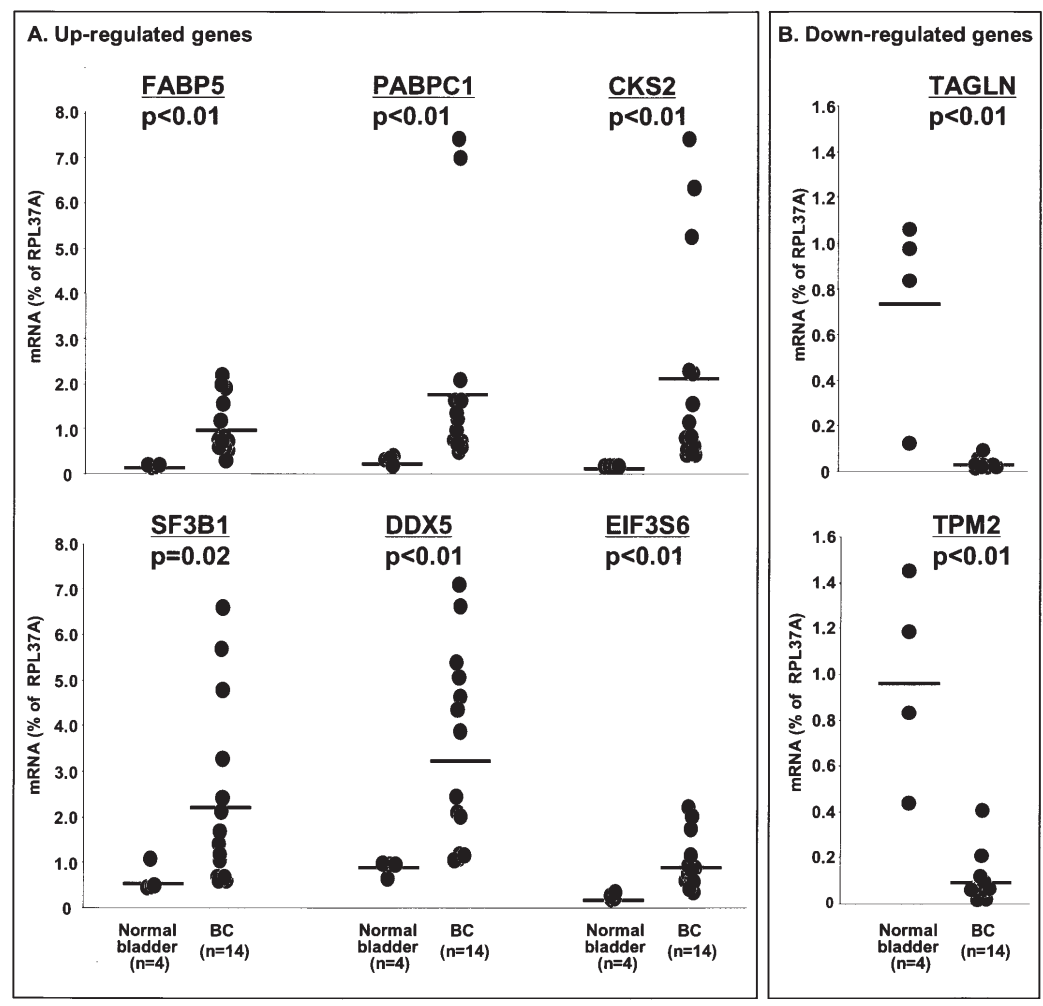

Figure 2. Comparison of the real-time PCR results for the 6 most highly up-regulated and the 2 most highly down-regulated genes and pathology (BC vs normal bladder). The relative mRNA expression examined was normalized to the amount of RPL37A. Statistical significance was determined by the MannWhitney U test.

Real-time RT-PCR verification of microarray results. We subjected the 6 most highly up-regulated genes and the 2 most highly down-regulated genes (Tables II and III) to realtime PCR and compared our results with those obtained for normal bladder ( $n=4)$ (Fig. 2). We found that the genes shown by microarray analysis as up-regulated did, indeed, show higher expression in $\mathrm{BC}$ than the normal bladder [FABP5: $0.11 \pm 0.01$ (normal bladder) vs 0.96 \pm 0.17 (BC), $\mathrm{p}<0.01 ; P A B P C 1: 0.24 \pm 0.05$ (normal bladder) vs $1.87 \pm 0.61$ (BC), $\mathrm{p}<0.01 ; C K S 2: 0.1 \pm 0.01$ (normal bladder) vs $2.1 \pm 0.64$ (BC), $\mathrm{p}<0.01 ; S F 3 B 1: 0.56 \pm 0.15$ (normal bladder) vs $2.27 \pm 0.54$ (BC), $\mathrm{p}=0.02 ; D D X 5: 0.82 \pm 0.08$ (normal bladder) vs $3.37 \pm 0.57(\mathrm{BC}), \mathrm{p}<0.01 ;$ EIF3S6: $0.19 \pm 0.35$ (normal bladder) vs $0.89 \pm 0.16(\mathrm{BC}), \mathrm{p}<0.01$; Fig. $2 \mathrm{~A}]$. The genes we identified as down-regulated manifested a lower expression in $\mathrm{BC}$ than the normal bladder [TAGLN: $0.74 \pm 0.21$ (normal bladder) vs $0.02 \pm 0.02(\mathrm{BC}), \mathrm{p}<0.01 ;$ TPM2: $0.96 \pm 0.22$ (normal bladder) vs $0.09 \pm 0.03(\mathrm{BC}), \mathrm{p}<0.01$; Fig. 2B]. Therefore, our microarray results reflect the actual mRNA levels of genes examined in our BC series.

Relationship between up-regulated genes and the BC stage. Using the 6 most highly up-regulated genes in Table II, we compared the mRNA expression level in superficial- and invasive BCs. Real-time PCR of the $14 \mathrm{BCs}$ subjected to microarray analysis demonstrated that $C K S 2$ was the only gene with significantly higher up-regulation in invasive than in superficial BC ( $\mathrm{p}=0.04)$ (Fig. 3). There was no difference between superficial- and invasive BC for the other 5 examined genes (Fig. 3). To confirm our results, we subjected all 21 BCs in this series to real-time PCR assay of CKS2 (Table I). As shown in Fig. 4, there was a high degree of difference between superficial- and invasive BC $[0.79 \pm 0.15$ (superficial) vs $2.78 \pm 0.52$ (invasive), $\mathrm{p}=0.001]$ and between the normal bladder and invasive BC $[0.12 \pm 0.01$ (normal bladder) vs $2.78 \pm 0.52$ (invasive $\mathrm{BC}$ ), $\mathrm{p}=0.001$ ]; there was less difference between the normal bladder and superficial $B C(p=0.005)$.

\section{Discussion}

We attempted to identify novel biomarkers for human BC by gene expression analysis of oligoarrays. We found that all 14 BCs subjected to microarray analysis shared 21 up- and 25 down-regulated genes; real-time RT-PCR analysis of the 6 most highly up-regulated and the 2 most highly downregulated genes confirmed our microarray results, indicating that they indeed reflect the actual mRNA levels of the genes examined in our BC series. Like others $(13,18)$, we used our microarray results to classify up- and down-regulated genes by their function. We found that $36 \%$ were involved in metabolism and $14 \%$ in transcription and processing. This may implicate them in the development and progression of $\mathrm{BC}$ (Fig. 1). The major function of our down-regulated genes was cell adhesion/surface- $(25 \%)$ or cytoskeleton/cell membrane-related $(21 \%)$, suggesting that BC cells acquire the ability to migrate via the down-regulation of these genes.

Assessment of the location of the 136 up-regulated genes showed that $11(8.1 \%)$ were on chromosome $7 \mathrm{p}, 10(7.4 \%)$ on $8 \mathrm{q}, 8(5.9 \%)$ on $12 \mathrm{q}, 7$ each $(5.1 \%)$ on $1 \mathrm{p}, 1 \mathrm{q}$, and $2 \mathrm{q}, 6$ each $(4.4 \%)$ on $15 q$ and $17 q, 5$ each $(3.7 \%)$ on $2 p$ and $20 q$, 


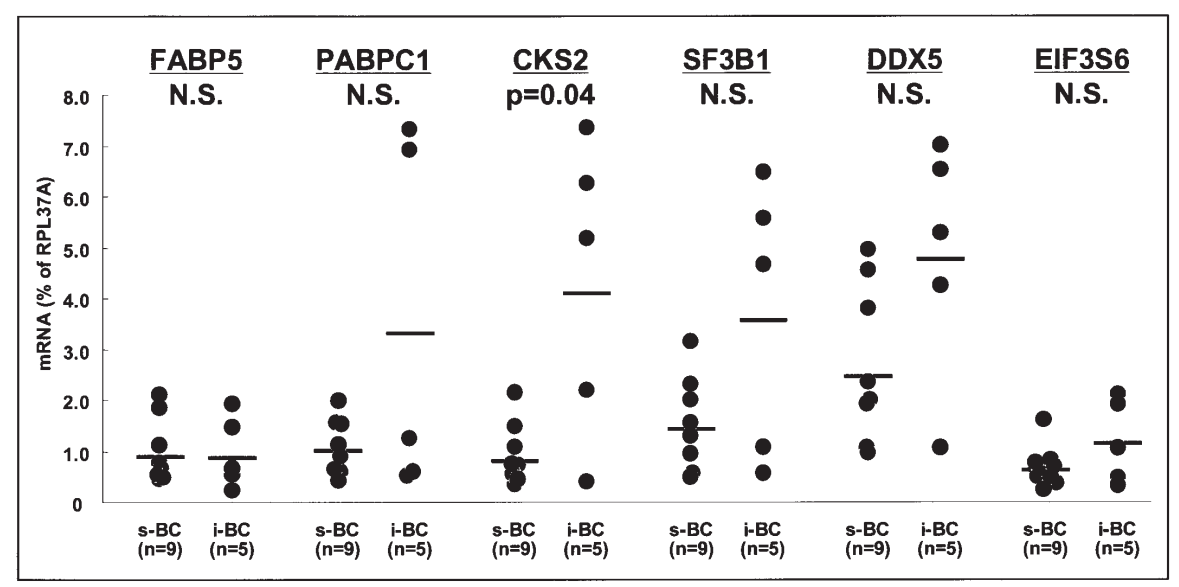

Figure 3. Comparison of the real-time PCR results for the 6 most highly up-regulated genes and the BC stage. Using samples from the 14 BCs subjected to microarray analysis, we found that $C K S 2$ was the only gene that was significantly up-regulated in invasive compared to superficial BC. The relative mRNA expression examined was normalized to the amount of RPL37A. Statistical significance was determined by the Mann-Whitney U test.

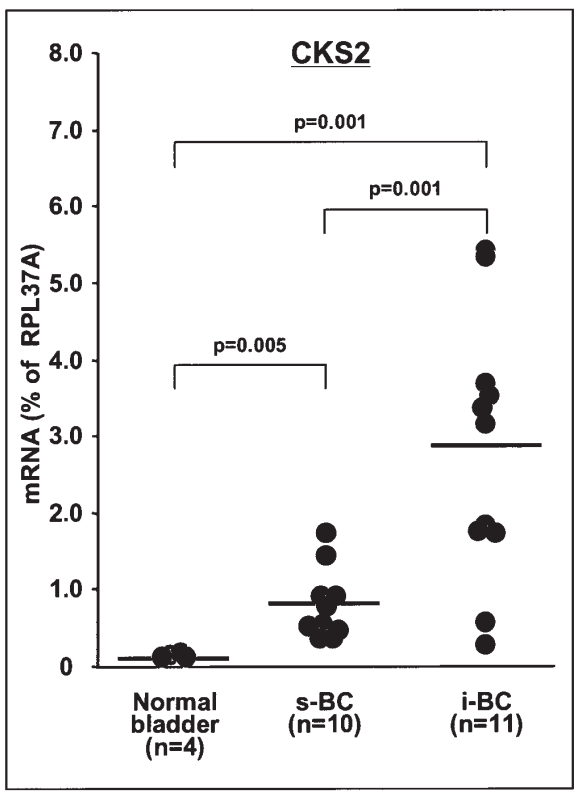

Figure 4. Real-time PCR analysis of CKS2 expression in tissue from normal bladders and 21 bladders with superficial or invasive cancer. There was a significant difference between superficial and invasive $B C(p=0.001)$, between normal bladders and bladders with invasive $\mathrm{BC}(\mathrm{p}=0.001)$, and between normal bladders and bladders with superficial $B C(p=0.005)$. The relative mRNA expression examined was normalized to the amount of RPL37A. Statistical significance was determined by the Bonferroni-adjusted Mann-Whitney U test.

and 4 each $(2.9 \%)$ on $5 q, 7 q, 14 q$, and 22q (Table II). Others (19-21) have shown by comparative genomic hybridization $(\mathrm{CGH})$ that the development and progression of $\mathrm{BC}$ is characterized by specific amplification involving chromosomes 1q, 2p, 3q, 5p, 6p, 8q, 11q, 17q, and 20q. We posit that several of the up-regulated genes in our BC series are associated with specific chromosomal amplification important in the development and progression of BC.

Among the 6 most highly up-regulated genes in our microarray analysis, FABP5, the gene with the highest level of up-regulation, carries fatty acids (FA) through the aqueous cellular environment and is involved in processes such as FA uptake, -transport, and -oxidation (22). This gene was also up-regulated in hepatocellular carcinoma cells compared with normal hepatocytes (23). PABPC1 is involved in translation and in the regulation of mRNA decay (24); it is significantly over-expressed in prostate cancer (25). CKS2, which encodes a cyclin kinase subunit of Cdc28/CDC2, is involved in cell-cycle progression from $\mathrm{G} 1$ to $\mathrm{S}$ and from $\mathrm{G} 2$ to $\mathrm{M}$; it is associated with lymphoid cell proliferation and its expression was increased in human acute lymphoblastic leukemia $(26,27)$. In addition, $C K S 2$ was expressed at significantly higher levels in colon cancer with than without liver metastasis (26). SF3B1 is absolutely required for premRNA splicing (28); its relationship with human cancer has not been reported. DDX5 (p68) is a prototypic member of the so-called DEAD box family of proteins (29) and an established RNA helicase (30); immunohistochemistry and Western blots showed it to be consistently over-expressed in colon cancer compared with matched normal tissues (31). EIF3S6 was first identified as a common virus insertion site in virally-induced mouse mammary tumors and preneoplastic lesions (32). Buttitta et al (33) reported that early-stage nonsmall cell lung cancer exhibited EIF3S6 mRNA levels higher than those observed in matching normal lung tissues. Ours is the first detailed investigation of these genes in human BC and our results suggest that they may be promising candidates for diagnostic BC biomarkers.

Bladder tumor antigen, the nuclear matrix protein 22, and the urinary bladder cancer antigen are clinically available diagnostic biomarkers for BC $(6,7)$. However, because of their insufficient sensitivity and specificity they cannot replace cystoscopy or cytology $(3,7)$ and patients with suspected BC continue to require painful cystoscopy. Microarray analysis of human $\mathrm{BC}$ has identified new cancerrelated genes, e.g. CKS2 (4,13-15), NPM1 (11,14), PMSA $(13)$, and PCNA $(4,13,15)$ and the results of gene profiling disclosed their association with tumor stage and progression and clinical outcomes. Although these studies have yielded useful insights into the molecular biology of human BC, they listed the genes without assessment of their value as 
biomarkers. We focused on the 6 genes that were most highly up-regulated in our microarray analysis and found that $C K S 2$ was uniquely and significantly up-regulated not only when we compared $\mathrm{BC}$ to normal bladder, but also when comparing invasive to superficial $\mathrm{BC}$. Therefore, the $C K S 2$ gene may be a biomarker not only for diagnosing but also for staging $\mathrm{BC}$. The difference in the $C K S 2$ expression level between invasive $\mathrm{BC}$ and the normal bladder was greater than between superficial BC and the normal bladder $(\mathrm{p}<0.001$ vs $\mathrm{p}<0.005$, Fig. 4), suggesting that $C K S 2$ expression may influence BC progression via cell-cycle progression. Studies are underway in our laboratory to elucidate the interactions between $C K S 2$ and related genes in $\mathrm{BC}$ and to assess the role of the down-regulated genes.

In conclusion, using oligonucleotide microarrays, we found that the CKS2 gene may be a biomarker for the diagnosis and staging of BC. Ours is the first report demonstrating that $C K S 2$ expression is strongly correlated with the progression of human BC. Our comprehensive expression profiling data of $\mathrm{BC}$ provide new insight into the molecular biology of $\mathrm{BC}$.

\section{Acknowledgements}

We thank Ms. M. Miyazaki for excellent laboratory assistance.

\section{References}

1. Jemal A, Murray T, Ward E, et al: Cancer statistics, 2005. CA Cancer J Clin 55: 10-30, 2005.

2. Al-Sukhun S and Hussain M: Molecular biology of transitional cell carcinoma. Crit Rev Oncol Hematol 47: 181-193, 2003.

3. Dyrskjot L, Thykjaer T, Kruhoffer M, et al: Identifying distinct classes of bladder carcinoma using microarrays. Nat Genet 33: 90-96, 2003.

4. Modlich O, Prisack HB, Pitschke G, et al: Identifying superficial, muscle-invasive, and metastasizing transitional cell carcinoma of the bladder: Use of cDNA array analysis of gene expression profiles. Clin Cancer Res 10: 3410-3421, 2004.

5. Knowles MA: What we could do now: Molecular pathology of bladder cancer. Mol Pathol 54: 215-221, 2001.

6. Giannopoulos A, Manousakas T, Gounari A, Constantinides C, Choremi-Papadopoulou $\mathrm{H}$ and Dimopoulos C: Comparative evaluation of the diagnostic performance of the BTA stat test, NMP22 and urinary bladder cancer antigen for primary and recurrent bladder tumors. J Urol 166: 470-475, 2001.

7. Mahnert B, Tauber S, Kriegmair M, et al: Measurements of complement factor H-related protein (BTA-TRAK assay) and nuclear matrix protein (NMP22 assay) - useful diagnostic tools in the diagnosis of urinary bladder cancer? Clin Chem Lab Med 41: 104-110, 2003.

8. Ogawa K, Utsunomiya T, Mimori K, et al: Genomic screens for genes upregulated by demethylation in colorectal cancer: possible usefulness for clinical application. Int J Oncol 27: 417-426, 2005

9. Nishidate T, Katagiri T, Lin ML, et al. Genome-wide geneexpression profiles of breast-cancer cells purified with laser microbeam microdissection: identification of genes associated with progression and metastasis. Int J Oncol 25: 797-819, 2004.

10. Lee BC, Cha K, Avraham S and Avraham HK: Microarray analysis of differentially expressed genes associated with human ovarian cancer. Int J Oncol 24: 847-851, 2004.

11. Sanchez-Carbayo M, Socci ND, Lozano JJ, et al: Gene discovery in bladder cancer progression using cDNA microarrays. Am J Pathol 163: 505-516, 2003.
12. Dyrskjot L, Kruhoffer M, Thykjaer T, et al: Gene expression in the urinary bladder: a common carcinoma in situ gene expression signature exists disregarding histopathological classification. Cancer Res 64: 4040-4048, 2004.

13. Thykjaer T, Workman C, Kruhoffer M, et al: Identification of gene expression patterns in superficial and invasive human bladder cancer. Cancer Res 61: 2492-2499, 2001.

14. Wild PJ, Herr A, Wissmann C, et al: Gene expression profiling of progressive papillary noninvasive carcinomas of the urinary bladder. Clin Cancer Res 11: 4415-4429, 2005.

15. Blaveri E, Simko JP, Korkola JE, et al: Bladder cancer outcome and subtype classification by gene expression. Clin Cancer Res 11: 4044-4055, 2005.

16. Sobin LH and Wittekind C: TNM classification of malignant tumours. In: International Union Against Cancer (UICC). 5th edition. Wiley-Liss Publications, New York, pp188-190, 1997.

17. The Japanese Urological Association and the Japanese Society of Pathology: General Rules for Clinical and Pathological Studies on Bladder Cancer. 2nd edition. Kanehara Publishing Co., Tokyo, p80, 1993

18. Arai M, Yokosuka O, Fukai K, et al: Gene expression profiles in liver regeneration with oval cell induction. Biochem Biophys Res Commun 317: 370-376, 2004.

19. Prat E, Bernues M, Caballin MR, Egozcue J, Gelabert A and Miro R: Detection of chromosomal imbalances in papillary bladder tumors by comparative genomic hybridization. Urology 57: 986-992, 2001.

20. Simon R, Burger H, Semjonow A, Hertle L, Terpe HJ and Bocker W: Patterns of chromosomal imbalances in muscle invasive bladder cancer. Int J Oncol 17: 1025-1029, 2000.

21. Richter J, Beffa L, Wagner U et al: Patterns of chromosomal imbalances in advanced urinary bladder cancer detected by comparative genomic hybridization. Am J Pathol 53: 1615-1621, 1998.

22. Kawamura T, Kanno R, Fujii H and Suzuki T: Expression of liver-type fatty-acid-binding protein, fatty acid synthase and vascular endothelial growth factor in human lung carcinoma. Pathobiology 72: 233-240, 2005.

23. Fujii K, Kondo T, Yokoo H, et al: Proteomic study of human hepatocellular carcinoma using two-dimensional difference gel electrophoresis with saturation cysteine dye. Proteomics 5: 1411-1422, 2005.

24. Kuhn U and Wahle E: Structure and function of poly(A) binding proteins. Biochim Biophys Acta 25: 67-84, 2004.

25. Van Duin M, van Marion R, Vissers K, et al: High-resolution array comparative genomic hybridization of chromosome arm 8q: evaluation of genetic progression markers for prostate cancer. Genes Chromosomes Cancer 44: 438-439. 2005.

26. Li M, Lin YM, Hasegawa S, et al: Genes associated with liver metastasis of colon cancer, identified by genome-wide cDNA microarray. Int J Oncol 24: 305-312, 2004.

27. Urbanowicz-Kachnowicz I, Baghdassarian N, Nakache C, et al: ckshs expression is linked to cell proliferation in normal and malignant human lymphoid cells. Int J Cancer 82: 98-104, 1999 .

28. Golas MM, Sander B, Will CL, et al: Molecular architecture of the multiprotein splicing factor SF3b. Science 300: 980-984, 2003.

29. Ford MJ, Anton IA and Lane DP: Nuclear protein with sequence homology to translation initiation factor eIF-4A. Nature 332: 736-78, 1988.

30. Hirling H, Scheffner M and Restle T: RNA helicase activity associated with the human p68 protein. Nature 339: 562-564, 1989.

31. Causevic M, Hislop RG, Kernohan NM, et al: Overexpression and poly-ubiquitylation of the DEAD-box RNA helicase p68 in colorectal tumours. Oncogene 20: 7734-7743, 2001.

32. Marchetti A, Buttitta F, Miyazaki S, et al: Int-6, a highly conserved, widely expressed gene, is mutated by mouse mammary tumor virus in mammary preneoplasia. J Virol 69: 1932-1938, 1995

33. Buttitta F, Martella C, Barassi F, et al: Int6 expression can predict survival in early-stage non-small cell lung cancer patients. Clin Cancer Res 11: 3198-3204, 2005. 